\title{
ERRATUM
}

\section{Onset of Coherent electromagnetic structures in the relativistic electron beam deuterium-tritium fuel interaction of fast ignition concern}

C. DEUTSCH,${ }^{1}$ A. BRET, ${ }^{2}$ M.-C. FIRPO,${ }^{3}$ L. GREMILLET ${ }^{4}$ E. LEFEBVRE, ${ }^{1}$ AND A. LIFSCHITZ ${ }^{1}$

${ }^{1}$ LPGP, Université Paris-Sud, Orsay, France

${ }^{2}$ ETSIL, Universitad Castilla-la Mancha, Cludad-Real, Spain

${ }^{3}$ LPTP, Ecole Polytechnique, Palaiseau, France

${ }^{4} \mathrm{CEN}$, Bruyéres-le-Châtel, France

(Volume 26, Number 2, 2008, pages 157-165; doi: 10.1017/S0263034608000189)

Because of a production error, we are here correcting Eq. (2)

$$
A=\left(\theta_{\mathrm{x}}^{\mathrm{p}}+p_{\mathrm{d}}^{\mathrm{p} 2} / m\right) / \theta_{\mathrm{y}}^{\mathrm{p}} \quad B=\left(\theta_{\mathrm{x}}^{\mathrm{b}}+p_{\mathrm{d}}^{\mathrm{b} 2} / m \gamma_{b}\right) / \theta_{\mathrm{y}}^{\mathrm{b}}
$$

